\title{
RELAÇÃO LIPASE/AMILASE NAS PANCREATITES AGUDAS DE CAUSA BILIAR E NAS PANCREATITES AGUDAS/CRÔNICAS AGUDIZADAS DE CAUSA ALCOÓLICA
}

\author{
Ricardo Custódio PACHECO' e Luiz Carlos Marques de OLIVEIRA $^{2}$
}

\begin{abstract}
RESUMO - Racional - Pancreatites agudas de causas alcoólica ou biliar podem necessitar de abordagens terapêuticas diferentes. Objetivo Verificar a validade da relação lipase/amilase em diferenciar as causas alcoólica ou biliar na pancreatite aguda/pancreatite crônica agudizada. Métodos - Foram avaliados nove pacientes com pancreatite aguda/pancreatite crônica agudizada alcoólica, todos homens, com idade média (desvio padrão) de 39,8 \pm 7,0 anos (grupo I) e 29 com pancreatite aguda biliar, sendo 8 homens e 21 mulheres, com idade média de 43,6 \pm 19,9 anos (grupo II). As amilasemias e lipasemias foram determinadas em pacientes com sintomatologia há, no máximo, 48 horas. A relação lipase/amilase foi calculada utilizando-se valores de amilasemia e lipasemia expressas como múltiplos de seus respectivos valores superiores de referência. Resultados - As médias das lipasemias $(4.814 \pm 3.670 \mathrm{U} / \mathrm{L})$ e amilasemias $(1.282 \pm 777 \mathrm{U} / \mathrm{L})$ no grupo I foram semelhantes às do grupo II ( $2.697 \pm 2.391$ e $1.878 \pm 1.319 \mathrm{U} / \mathrm{L}$, respectivamente), mas a média das relações lipase/amilase foi significantemente maior no grupo I $(4,4 \pm 3,6)$ do que no grupo II $(2,2 \pm 2,2)$. Relação lipase/amilase $>3$ foi significantemente mais freqüente no grupo I $(66,7 \%)$ do que no grupo II $(24,1 \%)$ e diferenciou os dois grupos com sensibilidade de $67 \%$ e especificidade de $76 \%$. Conclus $\tilde{\boldsymbol{e} e s}-1)$ as amilasemias e lipasemias não diferenciaram os dois grupos avaliados; 2) relação lipase/amilase >3 é mais freqüente na pancreatite aguda/pancreatite crônica agudizada alcoólica do que na pancreatite aguda biliar, e pode ser útil na diferenciação destas duas causas de pancreatite.

DESCRITORES - Amilases. Lipase. Pancreatite crônica. Pancreatite alcoólica.
\end{abstract}

\section{INTRODUÇÃO}

O diagnóstico de pancreatite aguda (PA) habitualmente é feito pelo quadro clínico de dor no andar superior do abdome, aumento dos níveis séricos de amilase e/ou lipase e confirmado por achados sugestivos em exames de imagem, como ao ultra-som (US) e/ou tomografia computadorizada (TC) de abdome. No entanto, estes exames podem não definir a causa da PA, exceto se forem observados cálculos biliares, principalmente na ausência de outras causas conhecidas de PA. Em nosso meio, as causas mais comuns de pancreatite aguda e pancreatite crônica agudizada (PCA) são a litíase biliar e o alcoolismo, respectivamente ${ }^{(16)}$. O reconhecimento da causa da pancreatite é importante para a abordagem terapêutica. $\mathrm{O}$ paciente com PA biliar (PAB) poderá, algumas vezes, se beneficiar com a esfincterotomia endoscópica e, sempre que possível, deverá ser submetido a colecistectomia antes da alta hospitalar para se evitar a recurrência de $\mathrm{PA}^{(15)}$. O paciente etilista necessitará de aconselhamento e/ou outra forma de tratamento para não voltar a beber. Essas diferenças nas abordagens terapêuticas justificam a busca por exames não-invasivos que possam auxiliar a história clínica e os exames de imagem no diagnóstico diferencial destas duas causas de pancreatite.

Para diferenciar a causa alcoólica de outras causas de PA, GUMASTE et al. ${ }^{(5)}$, em 1991, propuseram que, na admissão do paciente no hospital, a existência de uma relação lipase/amilase (L/A) maior do que 2 seria característica da PA alcoólica. Posteriormente, esses mesmos autores relataram que para o

Trabalho realizado no Hospital de Clínicas, Faculdade de Medicina, Universidade Federal de Uberlândia, Uberlândia, MG. Departamentos de ${ }^{1}$ Cirurgia e ${ }^{2}$ Clínica Médica da Faculdade de Medicina da Universidade Federal de Uberlândia, Uberlândia, MG. Apoio financeiro: Fundação de Assistência, Estudo e Pesquisa de Uberlândia (FAEPU).

Correspondência: Dr. Luiz Carlos Marques de Oliveira - Rua Gonçalves Dias, 455 - Bairro Tabajaras - 38400-288 - Uberlândia, MG. E-mail: oliveiralım@ufu.br 
diagnóstico da PA alcoólica a determinação da lipasemia seria melhor do que a da amilasemia ${ }^{(6)}$. Os estudos que foram realizados a seguir mostraram resultados conflitantes; enquanto em alguns se confirmou que a relação L/A era útil em diferenciar a causa alcoólica de outras causas $^{(7,10,14,23)}$, em outros ${ }^{(1,3,9,11,12,17,18,20,21)}$ foi observado que essa relação não apresentava alta sensibilidade e/ou especificidade, o que não justificaria o seu uso com essa finalidade.

O objetivo do presente estudo foi verificar a validade da relação L/A em diferenciar a causa alcoólica da biliar na PA/PCA, na admissão do paciente no serviço de emergência.

\section{MÉTODO}

Foram avaliados 38 pacientes com PA ou PCA, divididos em dois grupos. $\mathrm{O}$ grupo I foi composto por nove pacientes com PA alcoólica, todos do gênero masculino, com idade média e desvio padrão de $39,8 \pm 7,0$ anos, e intervalo de 30 a 47 anos. Desses, seis tinham evidências à TC de pancreatite crônica, e por isso tiveram o diagnóstico de PCA; dois não tinham evidências tomográficas de pancreatite crônica e o outro faleceu antes ser submetido a esse exame. O grupo II foi constituído por 29 pacientes com PAB, sendo 8 homens e 21 mulheres, com idade média e desvio padrão de 43,6 $\pm 19,9$ anos, e intervalo de 17 a 85 anos.

Os pacientes com sintomatologia no máximo há 48 horas, foram avaliados consecutivamente no Pronto Socorro do Hospital de Clínicas da Universidade Federal de Uberlândia, MG, e incluídos no estudo após as confirmações dos diagnósticos de PA e de sua causa. Os diagnósticos de PA ou de PCA basearam-se no quadro clínico de dor intensa no andar superior do abdome, com ou sem elevações dos níveis séricos de amilase e/ou de lipase, e foram sempre confirmados através de US e/ou TC de abdome ou durante laparotomia exploradora. Para o exame ultra-sonográfico de abdome foram utilizados aparelhos com análise espectral Doppler da marca Siemens modelo Sonoline Versa PRO ADC e transdutores de 3,5 MHz, e os critérios para o diagnóstico de PA se basearam no aumento volumétrico do pâncreas, diminuição de sua ecogenicidade e/ou presença de coleção líquida peripancreática ou retroperitonial ${ }^{(4)}$. As TC foram realizadas com aparelhos Somaton ART ou ARTX da marca Siemens e os critérios considerados para o diagnóstico de PA incluíam edema localizado ou difuso do pâncreas, irregularidade do contorno pancreático, borramento da gordura peripancreática, áreas heterogêneas com alterações da densidade no parênquima pancreático e presença de coleções líquidas peripancreáticas e/ou retroperitoniais ${ }^{(2)}$. Para o diagnóstico de PA durante a laparotomia, os critérios basearam-se, macroscopicamente, no aumento do volume e da consistência do pâncreas, com edema difuso ou localizado, acompanhado ou não de hemorragia, esteatonecrose peripancreática, necrose focal ou difusa do parênquima e presença de coleções líquidas peripancreáticas $\mathrm{e} / \mathrm{ou}$ retroperitoniais e/ou intraperitoniais ${ }^{(19)}$. O diagnóstico de pancreatite crônica ao exame tomográfico foi feito pela observação de atrofia glandular e/ou presença de calcificações e/ou dilatação e saculações dos ductos pancreáticos ${ }^{(8)}$.
A PA foi considerada como sendo de causa biliar quando se detectava colelitíase e/ou coledocolitíase nos exames de imagem ou durante laparotomia, e ausência de outras causas conhecidas de PA. O diagnóstico de PA/PCA alcoólica baseou-se na história de elevada ingestão etílica e ausência de litíase biliar ao US, além de outras causas de PA; todos os pacientes com PA/PCA alcoólica eram etilistas crônicos e ingeriam mais de $80 \mathrm{~g}$ de etanol por dia, por período maior que 6 anos. Nenhum dos pacientes tinha insuficiência renal e foram excluídos deste estudo aqueles em que se teve dúvida do diagnóstico de PA ou de sua causa.

As amostras de sangue para as determinações da amilasemia e da lipasemia foram coletadas na admissão do paciente no Pronto Socorro. As amilasemias foram determinadas utilizando-se o método colorimétrico enzimático com 2-cloro-4-nitrofenil$\beta$-D-maltoheptaoside (Cl-PNP-G7), com kits fabricados pela Roche Diagnostics GmbH, Mannhein, Alemanha, para aparelhos Cobas Integra. As determinações das lipasemias foram realizadas utilizado-se o método turbidimétrico com substrato de trioleina, com kits Unimate 3 LIP, fabricados pela Roche Diagnostics $\mathrm{GmbH}$, Mannhein, Alemanha, para aparelhos Cobas Mira.

Para o cálculo da relação L/A de cada um dos pacientes, inicialmente foram divididos os valores absolutos encontrados para a amilasemia e para a lipasemia pelo respectivo limite superior de referência (LSR) de cada um dos testes $(200 \mathrm{U} / \mathrm{L}$ e $190 \mathrm{U} / \mathrm{L}$, respectivamente). Quando os valores da amilasemia ou da lipasemia estavam dentro da faixa de normalidade, considerou-se que sua divisão pelo LSR era igual a um $(1,0)$. Posteriormente, o resultado encontrado para a lipasemia foi dividido por aquele da amilasemia, obtendo-se o resultado final da relação. De cada paciente ou de familiares foi obtido o consentimento informado para divulgação dos dados encontrados. Para o cálculo estatístico foram utilizados os testes não-paramétricos de Mann-Whitney e o exato de Fisher.

\section{RESULTADOS}

No grupo I, PA/PCA alcoólica, foram observados níveis de amilasemias que variaram entre 283 e $2.902 \mathrm{U} / \mathrm{L}$, com média e desvio padrão (DP) de $1.282 \pm 777 \mathrm{U} / \mathrm{L}$. Esses valores foram estatisticamente semelhantes aos do grupo II, PAB, no qual os níveis das amilasemias variaram entre 59 e $5.000 \mathrm{U} / \mathrm{L}$, com média (DP) de $1.878 \pm 1.319 \mathrm{U} / \mathrm{L}$. As lipasemias no grupo I, com valores mínimo e máximo de 37 e $10.900 \mathrm{U} / \mathrm{L}$, respectivamente, e média (DP) de $4.814 \pm 3.670 \mathrm{U} / \mathrm{L}$, também foram semelhantes às do grupo II, no qual as lipasemias variaram entre 94 e $9.180 \mathrm{U} / \mathrm{L}$, com média (DP) de $2.697 \pm 2.391 \mathrm{U} / \mathrm{L}$. A média (DP) das relações L/A do grupo I (4,4 $\pm 3,6)$ foi maior $(P<0,05)$ do que a do grupo II $(2,2 \pm 2,2)$ (Tabela 1). Relação L/A maior do que três ocorreu mais freqüentemente $(P<0,05 \%)$ no grupo I (em seis dos nove pacientes - 66,7\%) do que no grupo II (em 7 dos 29 pacientes $-24,1 \%$ ), e diferenciou o grupo I do grupo II com sensibilidade de $67 \%$, especificidade de $76 \%$, valor preditivo positivo de $46 \%$ e valor preditivo negativo de $88 \%$ (Tabela 2). Relação $L / A>3$ foi observada em quatro dos seis pacientes com diagnóstico de pancreatite crônica à TC de abdome e em dois nos quais este diagnóstico não foi confirmado. Quando se comparou as médias das amilasemias, lipasemias e 
TABELA 1. Amilasemia (U/L), lipasemia (U/L) e relação lipase/amilase (L/A) nos grupos I (pancreatite aguda/crônica agudizada alcoólica, $\mathrm{n}=9$ ) e II (pancreatite aguda biliar, $\mathrm{n}=29$ )

\begin{tabular}{ccccc}
\hline & & Amilasemia & Lipasemia & Relação L/A* \\
\hline \multirow{2}{*}{ Grupo I } & $\overline{\mathrm{X}}_{ \pm} \mathrm{DP}$ & $1.282 \pm 777$ & $4.814 \pm 3.670$ & $4,4 \pm 3,6$ \\
& Intervalo & $283-2.902$ & $37-10.900$ & $0,7-10,7$ \\
Grupo II & $\overline{\mathrm{X}}_{ \pm} \mathrm{DP}$ & $1.878 \pm 1.319$ & $2.697 \pm 2.391$ & $2,2 \pm 2,2$ \\
& Intervalo & $59-5.000$ & $94-9.180$ & $0,1-8,8$ \\
\hline
\end{tabular}

* $P<0,05$ (teste de Mann-Whitney), grupo I $>$ grupo I

$\mathrm{Z}=$ média

$X=$ media
$D P=$ desvio padrão

valores normais das enzimas: amilasemia até $200 \mathrm{U} / \mathrm{L}$ e lipasemia até $190 \mathrm{U} / \mathrm{L}$

TABELA 2. Validade da relação lipase/amilase (L/A) maior do que 3 em diferenciar a pancreatite aguda/crônica agudizada alcoólica $(n=9)$ da biliar $(\mathrm{n}=29)$

\begin{tabular}{cccc}
\hline & Alcoólica & Biliar & $\boldsymbol{P}^{*}$ \\
\hline $\mathrm{L} / \mathrm{A}>3$ & $6 / 9(66,7 \%)$ & $7 / 29(24,1 \%)$ & $<0,05$ \\
\hline${ }^{*}$ Teste exato de Fisher & & & \\
Sensibilidade $=67 \%$ & & & \\
Especificidade $=77 \%$ & & & \\
Valor preditivo positivo $=46 \%$ & & \\
Valor preditivo negativo $=88 \%$ & &
\end{tabular}

relação L/A entre os gêneros masculino (1.298 $\pm 824 \mathrm{U} / \mathrm{L}, 2.618$ $\pm 2.682 \mathrm{U} / \mathrm{L}$ e $2,4 \pm 1,8$; respectivamente) e feminino (2.100 \pm $1.419 \mathrm{U} / \mathrm{L}, 2.728 \pm 2.341 \mathrm{U} / \mathrm{L}$ e $2,2 \pm 2,4$; respectivamente), no grupo II, foi observado que não havia diferença estatisticamente significante entre elas.

\section{DISCUSSÃO}

Os resultados do presente estudo mostram que não foi possível diferenciar as causas alcoólica ou biliar da PA com relação aos níveis das amilasemias ou lipasemias. A média das amilasemias do grupo com $\mathrm{PAB}$ foi discretamente superior àquela do grupo com PA/PCA alcoólica, enquanto que, a média das lipasemias do grupo com PA/PCA alcoólica foi quase o dobro daquela observada no grupo com PAB. Apesar disso, a diferença entre as médias das lipasemias nos dois grupos não foi estatisticamente significante, provavelmente, devido à variabilidade observada entre os valores individuais desta enzima, o que pode ser percebido pelo grande desvio padrão encontrado em ambos os grupos. Estes resultados são semelhantes àqueles encontrados por GUMASTE et al. ${ }^{(6)}$, quando descreveram que a determinação da lipasemia é melhor do que a da amilasemia para o diagnóstico da PA alcoólica. No entanto, também já foi descrito que na PA alcoólica o aumento da amilasemia é significantemente mais baixo do que naquelas de outras causas ${ }^{(5,}$ 23) e, ainda, que tanto a amilasemia quanto a lipasemia estariam mais elevadas na PA de causa não-alcoólica do que naquelas de causa alcoólica ${ }^{(9,18)}$ e isto, provavelmente, seria decorrente de lesões crônicas no pâncreas ocasionadas pelo álcool.

Desde o estudo inicial de GUMASTE et al.(5), no qual observaram que uma relação $\mathrm{L} / \mathrm{A}>2$ seria útil em diferenciar a causa alcoólica da não-alcoólica na PA, com altas sensibilidade (91\%), especificidade (78\%) e valor preditivo positivo (85\%), outras investigações foram conduzidas nesse sentido e apresentaram resultados conflitantes. Enquanto em algumas se confirmou a importância desta relação ${ }^{(7,14,23)}$, em outras foram encontradas baixas sensibilidade e/ou especificidade ${ }^{(1,3,9,11,12,17,18,20,21)}$ e concluíram que a relação L/A não seria útil na diferenciação das causas das pancreatites agudas. $\operatorname{GUMASTE}^{(7)}$, revendo alguns trabalhos, descreveu que apesar da relação L/A não ser um teste padrão-ouro na diferenciação da causa da PA, conclui que uma relação L/A $<2$ seria improvável indicar PA alcoólica, sendo sugestiva de causa não-alcoólica; relação L/A $>3$ mais provavelmente se associaria à causa alcoólica e uma taxa entre 2 e 3 estaria em faixa de não especificidade. Outros investigadores observaram que a relação L/A poderia ser útil na diferenciação das causas da PA desde que utilizada em conjunto com alterações concomitantes de outros exames laboratoriais, como a aspartato aminotransferase (AST) ${ }^{(10)}$, ou ainda, AST, alanina-aminotransferase (ALT), fosfatase alcalina e volume corpuscular médio das hemácias, em um sistema de escore ${ }^{(22)}$.

No presente estudo foi observado que tanto a média das relações $\mathrm{L} / \mathrm{A}$, quanto a freqüência da relação $\mathrm{L} / \mathrm{A}>3$ foram significantemente maiores no grupo I do que no grupo II, no entanto, a sensibilidade (67\%), a especificidade (76\%) e o valor preditivo positivo $(46 \%)$ não foram tão altos quantos aqueles descritos por GUMASTE et al. ${ }^{(5)}$.

As explicações para esta maior freqüência da relação $\mathrm{L} / \mathrm{A}>3$ na PA/PCA alcoólica do que na PAB, e ainda, para os diferentes resultados encontrados na literatura, não estão claras. No estudo de GUMASTE et al.$^{(5)}$ todos os pacientes com PA alcoólica eram homens e o grupo com PA não-alcoólica era formado somente por mulheres, o que levou alguns autores a supor que a relação L/A diferenciasse PA entre homens e mulheres. No entanto, os resultados do presente estudo mostram que as médias das amilasemias, das lipasemias e das relações L/A foram semelhantes entre os gêneros, masculino e feminino, o que já havia sido observado em outros trabalhos ${ }^{(1,11,18)} \mathrm{e}$, portanto, esta não seria a razão para as diferenças descritas anteriormente. Também já foi descrito que a relação L/A não diferencia PA leve de PA grave, assim como não é influenciada pelas idades dos pacientes ${ }^{(18)}$.

Outro fator a ser considerado é o intervalo de tempo entre o início dos sintomas da PA e o momento da coleta de sangue para as determinações da amilasemia e da lipasemia. Na evolução dessa doença, a amilasemia volta ao nível normal mais rapidamente do que a lipasemia ${ }^{(16)}$, portanto, a coleta de sangue para as determinações dessas enzimas em fases mais tardias, no curso da PA, pode ser motivo para se encontrar elevada relação L/A. Para se evitar esse viés, somente foram avaliados pacientes que tinham sintomatologia há menos de 48 horas, cuidado que também foi tomado na maioria dos trabalhos citados anteriormente.

Seis dos nove pacientes com PA alcoólica tinham sinais sugestivos de pancreatite crônica à TC, ou seja, a PA foi provavelmente agudização de um processo crônico; dois pacientes não tinham sinais tomográficos de pancreatite crônica e um outro não foi avaliado posteriormente porque faleceu durante a internação. Esses achados são concordantes com aqueles descritos em estudo recente ${ }^{(13)}$, no qual se observou que a PA alcoólica se desenvolve em pâncreas já comprometido com pancreatite crônica. Acredita-se que as fibroses e a diminuição do tecido acinar, decorrentes das alterações crônicas do pâncreas ocasionadas pelo álcool, possam por mecanismos fisiopatológicos ainda não esclarecidos, prejudicar 
mais a síntese pancreática da amilase do que a da lipase ${ }^{(1)}$. Isso poderia ser melhor observado durante o episódio de agudização da pancreatite crônica, e ser o motivo pelo qual, em alguns estudos, a relação $\mathrm{L} / \mathrm{A}>3$ foi mais freqüente em pacientes com PA alcoólica do que naqueles com PA biliar. Nesse sentido, o fato de dois dos pacientes do grupo I, na presente casuística, não possuírem sinais de pancreatite crônica à $\mathrm{TC}$, mas terem relação $\mathrm{L} / \mathrm{A}>3$, pode ser indício de que eles já tenham tido lesões pancreáticas crônicas decorrentes do alcoolismo. As alterações crônicas do pâncreas produzidas pelo álcool poderiam explicar, inclusive, porque ocorrem aumentos discretos da amilasemia e da lipasemia em alguns grupos de pacientes com PA alcoólica ${ }^{(17)}$. No entanto, nos trabalhos anteriores não há descrições de que os pacientes com PA alcoólica tivessem pancreatite crônica, como foi agora observado neste estudo. Para se comprovar esta hipótese, futuras investigações serão necessárias, avaliando inclusive pacientes com pancreatite crônica de outras causas.

\section{CONCLUSÕES}

Os resultados do presente estudo permitem concluir que, na população estudada: 1) não foi possível diferenciar PAB de PA/PCA alcoólica em relação às amilasemias e às lipasemias; 2) a relação $\mathrm{L} / \mathrm{A}>3$ é mais freqüente em pacientes com $\mathrm{PA} /$ PCA alcoólica do que naqueles com PAB, e pode ser útil na diferenciação dessas duas causas de pancreatite.

Pacheco RC, Oliveira LCM. Lipase/amylase ratio in biliary acute pancreatitis and alcoholic acute/acutized chronic pancreatitis. Arq Gastroenterol. 2007;44(1):35-8.

ABSTRACT - Background - Alcoholic or biliary acute pancreatitis may need different therapeutic approaches. Aim - Assessing the validity of lipase/amylase ratio in differentiating biliary from alcoholic acute pancreatitis/acutized chronic pancreatitis. Methods - Nine male patients (mean age and standard deviation: $39.8 \pm 7.0$ years) with alcoholic acute pancreatitis/acutized chronic pancreatitis (group I) and 29 patients, 8 male and 21 female (mean age: $43.6 \pm 19.9$ years), with biliary acute pancreatitis (group II) were evaluated. Serum lipase and amylase levels were measured in patients with symptoms for no more than 48 hours. The lipase/amylase ratio was calculated based on serum lipase and amylase levels and expressed as multiples of their respective superior reference values. Results - Mean levels of serum lipase (4,814 $\pm 3,670 \mathrm{U} / \mathrm{L})$ and amylase $(1,282 \pm 777 \mathrm{U} / \mathrm{L})$ in patients of group I were comparable to group II $(2,697 \pm 2,391$ and $1,878 \pm 1,319 \mathrm{U} / \mathrm{L}$, respectively), but the mean lipase/amylase ratio was significantly higher in group I (4.4 \pm 3.6$)$ than in group II (2.2 \pm 2.2 ). Lipase/amylase ratio $>3$ occurred at significantly higher proportions in patients of group I (66.7\%) than of group II ( $24.1 \%)$, differentiating the two groups with sensitivity of $67 \%$ and specificity of $76 \%$. Conclusions - 1) Amylase and lipase serum levels did not differ in the two groups evaluated; 2 ) the lipase/amylase ratio $>3$ was more often seen in alcoholic acute pancreatitis/acutized chronic pancreatitis than biliary acute pancreatitis, and it may be useful in differentiating these two causes of pancreatitis.

HEADINGS - Amylases. Lipase. Pancreatitis, chronic. Pancreatitis, alcoholic.

\section{REFERÊNCIAS}

1. Ansari E, Talenti DA, Scopelliti JA, Saadat JM, Zehr BD. Serum lipase and amylase ratio in acute alcoholic and nonalcoholic pancreatitis by using Dupont ACA discrete clinical analyzer. Dig Dis Sci. 1996;41:1823-7.

2. Balthazar EJ. CT diagnosis and staging of acute pancreatitis. Radiol Clin North Am. 1989;27:19-37.

3. Deltenre P, Ghilain J.-M, Maisin J.-M, Schapira M, Henrion J, Heller FR. Le rappor L/A permet-il d'identifier les pancréatites aiguës alcooliques? Acta Gastroentero Belg. 1995;58:222-9.

4. Doust BD, Pearce JD. Gray-scale ultrasonic properties of the normal and inflamed pancreas. Radiology. 1976;120:653-7.

5. Gumaste VV, Dave PB, Weissman D, Messer J. Lipase/amylase ratio. A new index that distinguishes acute episodes of alcoholic from nonalcoholic acute pancreatitis Gastroenterology. 1991;101:1361-6.

6. Gumaste V, Dave P, Sereny G. Serum lipase: a better test to diagnose acute alcoholic pancreatitis. Am J Med. 1992;92:239-42

7. Gumaste VV. Lipase/amylase ratio: a review. Pancreas. 1995;10:210-2.

8. Haaga JR. The pancreas. In: Haaga JR, Lanzieri CF, Gilkeson RC, editors. CT and MR imaging of the whole body. 4th ed. St. Louis: Mosby; 2003. v. 2, p.1395-486.

9. Jaakkola M, Sillanaukee P, Löf K, Koivula T, Nordback I. Blood tests for detection of alcoholic cause of acute pancreatitis. Lancet. 1994;343:1328-9.

10. Kazmierczak SC, Catrou PG, Van Lente F. Enzymatic markers of gallstone-induced pancreatitis identified by ROC curve analysis, discriminant analysis, logistic regression, likehood ratios, and information theory. Clin Chem. 1995;41:523-31.

11. King LG, Seelig CB, Ranney JE. The lipase to amylase ratio in acute pancreatitis Am J Gastroenterol. 1995;90:67-9.

12. Laurent-Puig P, Boutron A, Briantais MJ, Vahedi K, Fritsch J, Choury AD, Pelletier G, Buffet C. Lipase/amylase ratio in pancreatitis: an etiologic index? Gastroenterology. 1992;103:353-4
13. Migliori M, Manca M, Santini D, Pezzilli R, Gullo L. Does acute alcoholic pancreatitis precede the chronic form or is the opposite true?: a histological study. J Clin Gastroenterol. 2004;38:272-5.

14. Moster SG, Herbstman D, Kniaz JL, Lurwick DR, Efrusy ME. Use of lipase:amylase ratio (LA ratio) in distinguishing alcoholic vs. gallstone causes of acute pancreatitis [abstract]. Am J Gastroenterol. 1993;88:1536.

15. Nealon WH, Bawduniak J, Walser EM. Appropriate timing of cholecystectomy in patients who present with moderate to severe gallstone-associated acute pancreatitis with peripancreatic fluid collections. Ann Surg. 2004;239:741-51.

16. Pacheco RC, Nishioka SA, Oliveira LCM. Validade da amilasemia e da lipasemia no diagnóstico diferencial entre pancreatite aguda/crônica agudizada e outras causas de dor abdominal aguda. Arq Gastroenterol. 2003;40:233-8.

17. Pezzilli R, Billi P, Miglioli M, Gullo L. Serum amylase and lipase concentrations and lipase/amylase ratio in assessment of etiology and severity of acute pancreatitis. Dig Dis Sci. 1993;38:1265-9.

18. Pezzilli R, Billi P, Barakat B, Miglio F. Lipase-amylase ratio does not determine the etiology of acute pancreatitis. J Clin Gastroenterol. 1998;26:34-8.

19. Raia AA, Machado MCC. Pancreatite aguda. In: Raia AA, Zerbini EJ, editores. Clínica cirúrgica Alípio Corrêa Netto. $4^{\mathrm{a}}$ ed. São Paulo: Sarvier; 1988. v.4, p.902-13.

20. Sadowski DC, Sutherland LR. The lipase/amylase ratio: sensitive but not specific. Gastroenterology. 1992;103:352-3.

21. Sadowski DC, Todd K, Sutherland LR. Biochemical models as early predictors of the etiology of acute pancreatitis. Dig Dis Sci. 1993;38:637-43.

22. Stimac $\mathrm{D}$, Lenac $\mathrm{T}$, Marusic $Z$. A scoring system for early differentiation of the etiology of acute pancreatitis. Scand J Gastroenterol. 1998;33:209-11.

23. Tenner SM, Steinberg W. The admission serum lipase:amylase ratio differentiates alcoholic from nonalcoholic acute pancreatitis. Am J Gastroenterol. 1992;87:1755-8.

Recebido em 17/1/2006. Aprovado em 31/5/2006. 